\title{
Comparativa en las pautas de movilidad entre estaciones periférica y céntrica de alta velocidad: los casos de Alicante y Villena
}

\author{
David Bautista Rodríguez \\ Ingeniero de Caminos, Canales y Puertos
}

Armando Ortuño Padilla

Profesor contratado doctor del Departamento de Edificación y Urbanismo, Universidad de Alicante, España

\section{RESUMEN}

En esta ponencia se tratan de evaluar las diferencias en los patrones de movilidad de los viajeros en una estación de alta velocidad periférica -Villena- y otra céntrica -Alicante- tras la puesta en servicio de la alta velocidad correspondiente al corredor Madrid - Levante en junio de 2013.

\section{INTRODUCCIÓN}

La provincia de Alicante, por su extensión costera con cerca de $250 \mathrm{~km}$ y su cálido clima genera una gran atracción de turistas principalmente procedentes de Madrid y sus cercanías aunque también cabe destacar el norte de España. Además de esto, hay que señalar es una de las más pobladas de España y que entre sus municipios destacan tanto los de costa e interior, caracterizados también por su intensa actividad industrial.

En lo que se refiere a los servicios de alta velocidad, la línea Madrid - Levante se inauguró en junio de 2013 tanto con trenes directos como con paradas intermedias en Cuenca, Albacete y Villena, en total, 18 servicios en días laborables ( 9 por sentido). Algunos estudios sobre demanda de este corredor predicen que esta línea será una de las más concurridas en el ámbito nacional. En la puesta en marcha de la línea, el viaje de 486 km entre Alicante y Madrid se recorría en 2,5 horas llegando a reducirse en el primer año a poco más de 2 horas. En los servicios anteriores, el trayecto en ferrocarril convencional duraba poco más de 3 horas, llegando a ahorrar más de una hora. Por otro lado, en el primer año de funcionamiento se transportaron 1.732 .000 viajeros (renfe, 2014). 


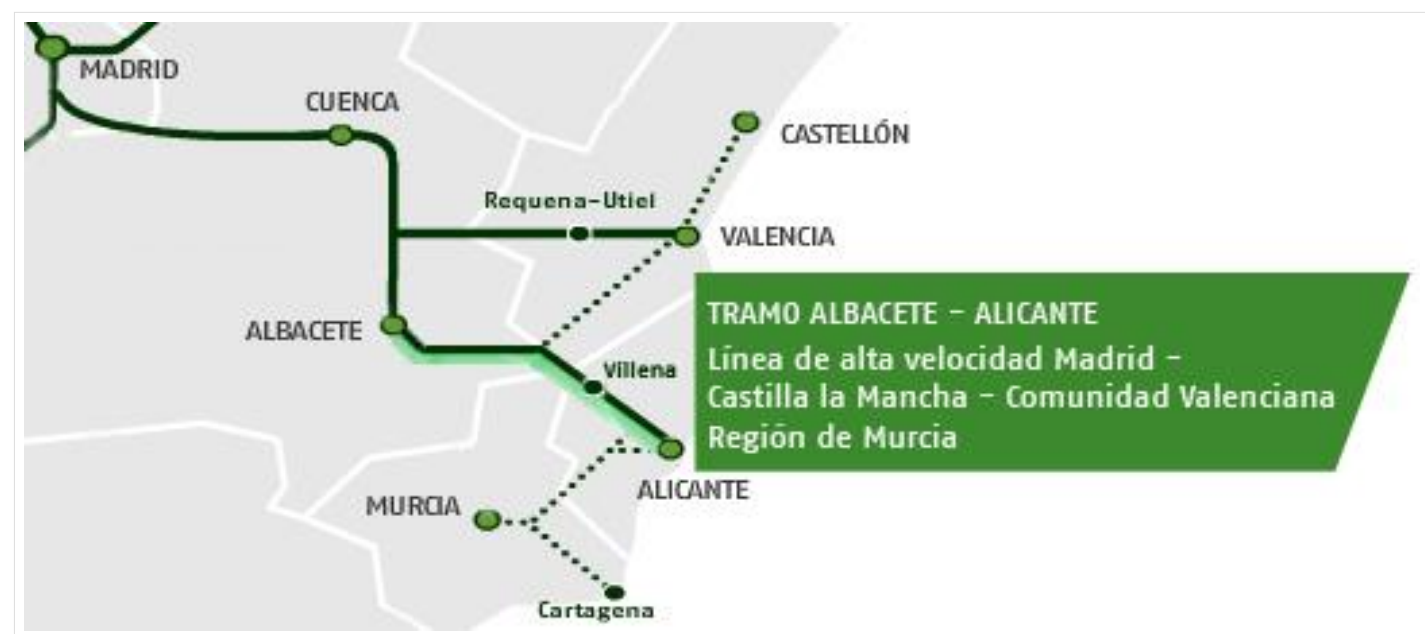

Fig. 1 - Línea de Alta Velocidad Madrid - Levante.

\subsection{Metodología de la encuesta.}

Con objeto de estimar el perfil socioeconómico de los viajeros, se realizaron 1.963 encuestas en la estación de Alicante y 911 en la estación de Villena distribuidas en las estaciones de invierno, primavera, verano y otoño, garantizando una significación estadística del 95\%. En la realización de encuestas, también se tuvo en cuenta la diferenciación entre día laboral o fin de semana, siempre sin influencia de días festivos y cubriéndose todos los servicios diarios. Así mismo, se diferenció entre el viajero con destino provincia de Alicante y con destino Madrid u otros destinos peninsulares.

\section{RESULTADOS DE LA ENCUESTA}

Aunque la encuesta era muy extensa, se ha estimado conveniente recoger exclusivamente los resultados en que más relevantes han sido las diferencias en ambas estaciones: Alicante y Villena.

\subsection{Perfil socioeconómico}

En lo que se refiere al perfil socioeconómico, una de las variables a tener en cuenta es el nivel de renta, se puede apreciar que en ambas estaciones cerca del $75 \%$ de los viajeros están en un rango de 1.001 a 3.000 euros mensuales siendo para el caso de Villena notablemente más elevado.

En cuanto a nivel educativo los resultados arrojan que, siendo el predominante en ambos casos (cercano al 50\%), en Villena el nivel universitario es mayor con un 58\%.

En el caso del rango de edad es interesante estudiar el caso desagregado por estación climática del año, ya que para la estación de Alicante existe una variabilidad acentuada en verano mientras que para Villena existe cierta constancia. Para el caso de Alicante hay que señalar que en las épocas de invierno y primavera el perfil con más de 65 años ronda el $50 \%$ de los viajeros. 

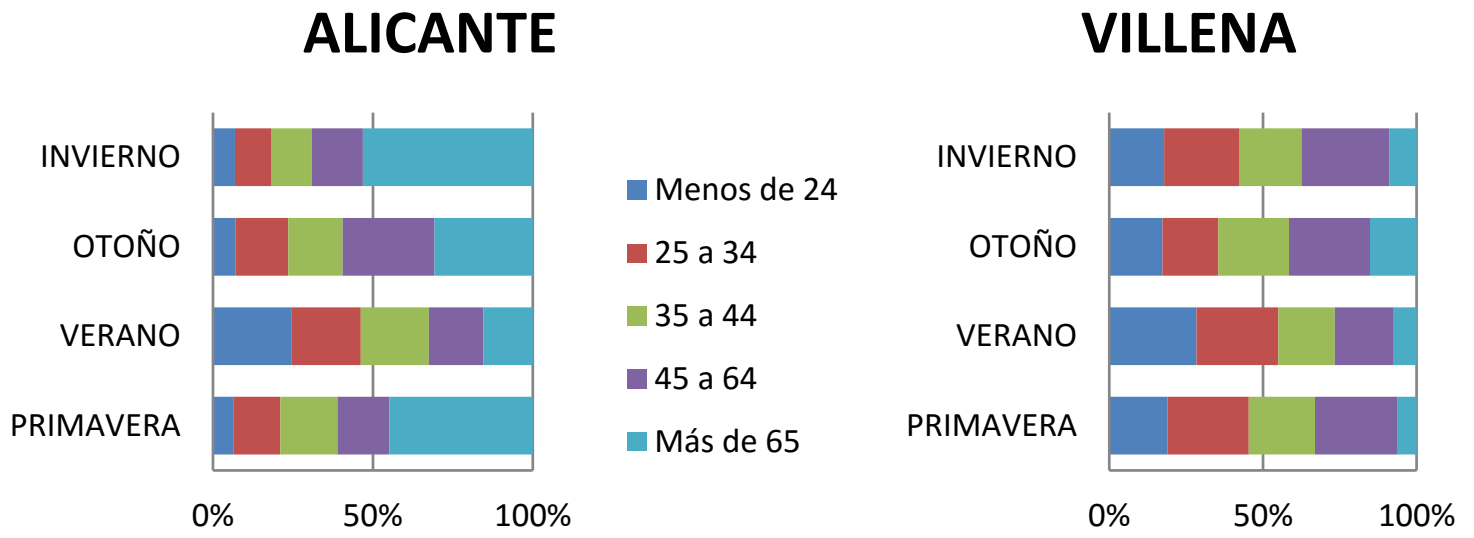

Fig. 2 y 3 - rango de edad de los viajeros de la estación de Alicante y Villena por estación del año.

\subsection{Características de movilidad}

Referente a características de la zona de influencia por captación de viajeros se consideran las características de accesibilidad, la tipología de los billetes o competencia entre modos de transporte. En cuanto a este último, para ambas estaciones cerca de un $30 \%$ de los viajeros consideraron usar otro modo de transporte (fig. 4) y de éstos, el $50 \%$ consideraron el vehículo privado como modo alternativo, siendo más acentuado en el caso de Villena (fig. 5). El siguiente modo más notable con cerca de un $20 \%$ para Villena y del $25 \%$ para Alicante es el avión.
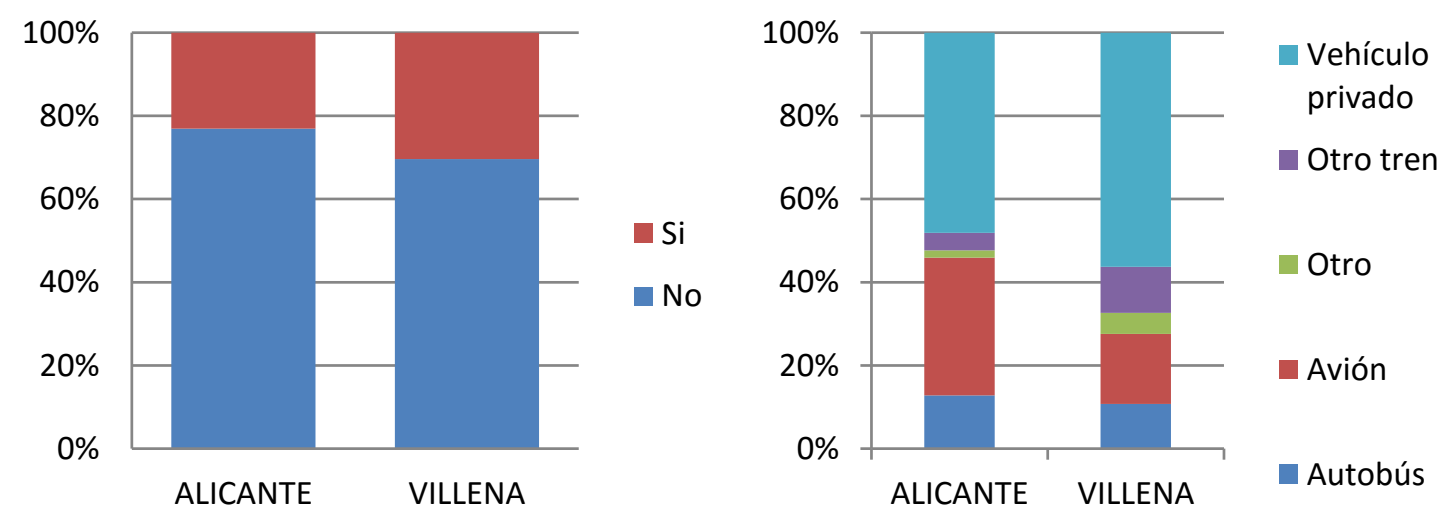

Fig. 4 y 5 - consideración sobre el uso de otro modo y qué modo por estación.

Analizando el tipo de billete contratado, en ambas estaciones aparecen los mismos resultados encontrando la diferencia en el destino que motiva el viaje del cliente. Cuando el viajero tiene el origen en las estaciones de Alicante o Villena, más del $75 \%$ de los viajeros escogen billete turista mientras que en el caso de que el destino sea Alicante o Villena se acentúa rozando el $90 \%$.

Para finalizar con este apartado, si se consideran las condiciones de acceso, previamente 
hay que resaltar la centralidad de la estación de Alicante, situada a escasos metros de los ejes comerciales de la ciudad y con buena conectividad con los servicios de transporte público, mientras que la estación de Villena dista más de $4 \mathrm{~km}$ del núcleo y del acceso más cercano a la autovía, a lo que se suma la inexistencia de autobuses o autocares que ofrezcan servicio.

Bajo estas premisas, los datos son consistentes con estas condiciones de accesibilidad, mostrando que para la estación de Alicante cerca del 25\% de los viajeros están a menos de 10 minutos mientras que para el caso de Villena éstos apenas llegan al 10\% (fig. 6). En estos resultados también destaca un grueso de viajeros de Villena cercano al 30\% que tardan en acceder a la estación en torno a los 30 minutos, mientras que para el caso de Alicante se reparte equitativamente en 10, 15 y 20 minutos el 50\% de los viajeros.

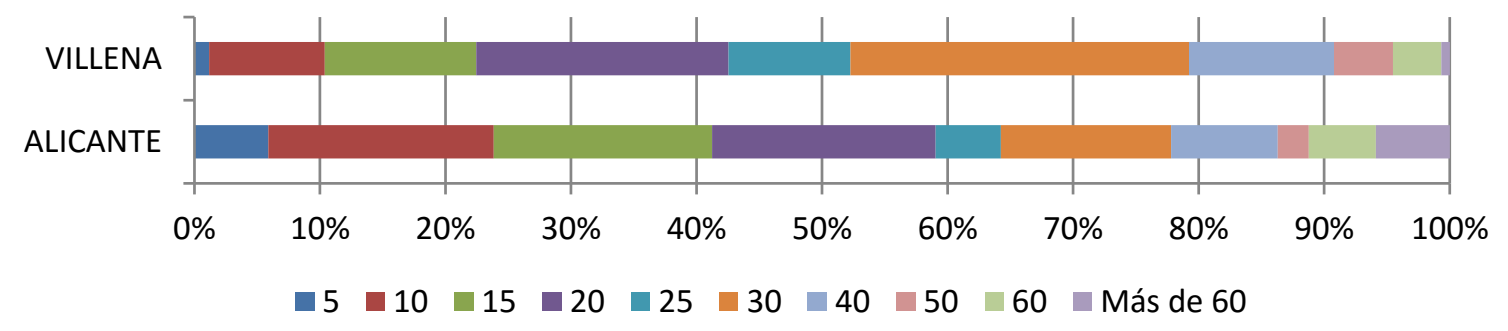

Fig. 6: tiempo de acceso a las estaciones de Alicante y Villena.

En el estudio de los modos de acceso, en el caso de Villena el vehículo privado es el más utilizado con más de un $80 \%$ seguido del taxi, que supone cerca del 15\%. En la estación de Alicante, la diferencia recae en la presencia de modos de transporte público (excluyendo el taxi) que suponen más de un $25 \%$ de los viajeros que acceden, restándole protagonismo principalmente al vehículo privado (propio o familiar).

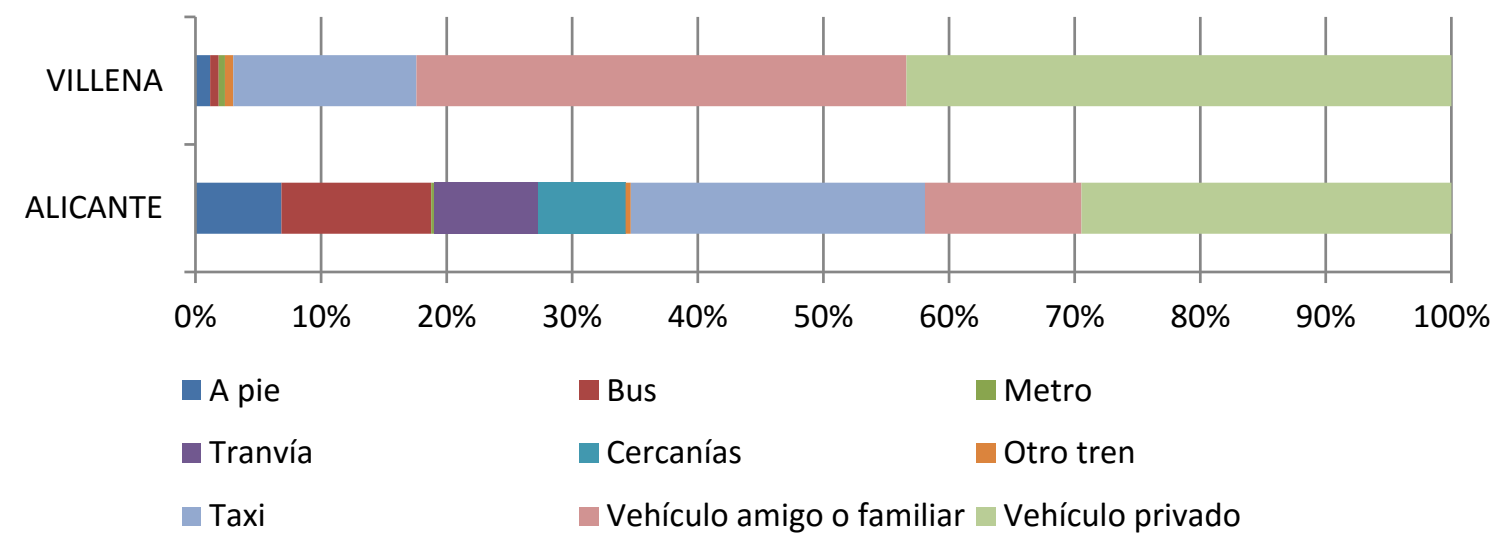

Fig. 7: modo de acceso a la estación de Alicante y Villena.

En relación a lo anterior, también hay que señalar el tiempo de estancia en la estación, los viajeros que acuden a la estación de Villena no permanecen un tiempo mayor a los 20 
minutos mientras que para el caso de Alicante sólo un $20 \%$ de los viajeros pasa 20 minutos o menos. Además, cerca de un $20 \%$ de los viajeros llegan a estar más de 60 minutos.

\subsection{Evaluación de los destinos y motivos de viaje}

En el caso de que los viajeros inicien su viaje en la provincia de Alicante, los destinos, ya sean desde la estación de Alicante o Villena son muy similares, siendo Madrid claramente el preferido, seguido de otros núcleos relativamente cercanos a la capital con un sesgo hacia los del norte peninsular. En las figuras 8 y 9 se muestran los municipios destino a los que se dirigen un mínimo del $0,5 \%$ de los encuestados.

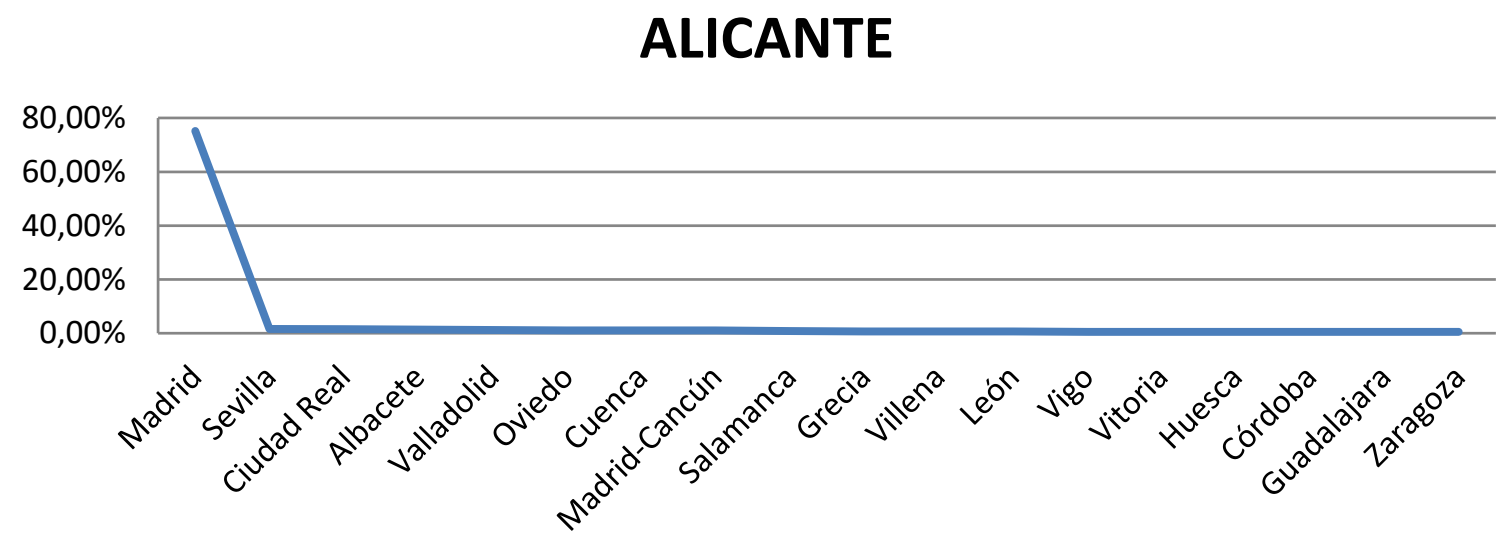

\section{VILLENA}

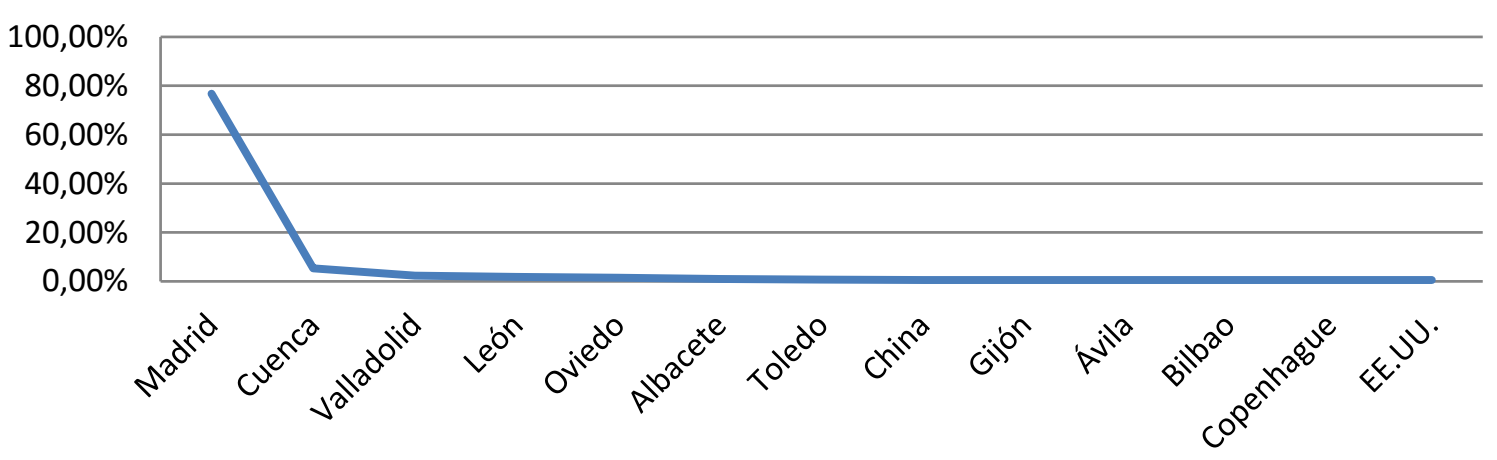

Fig. 8 y 9: destinos de viajeros con origen la provincia de Alicante por estación.

En el lado opuesto, cuando los viajeros tienen como destino la provincia de Alicante sí se aprecia una diferencia clara entre estaciones. Para el caso de la estación de Alicante, los destinos son principalmente núcleos costeros con actividad turística significativa, mientras que para el caso de Villena los destinos son municipios cercanos y pertenecientes su área de influencia (interior de la Provincia de Alicante). En las figuras 10 y 11 se muestran los municipios destino a los que se dirigen un mínimo del 0,5\% de los encuestados. 


\section{ALICANTE}

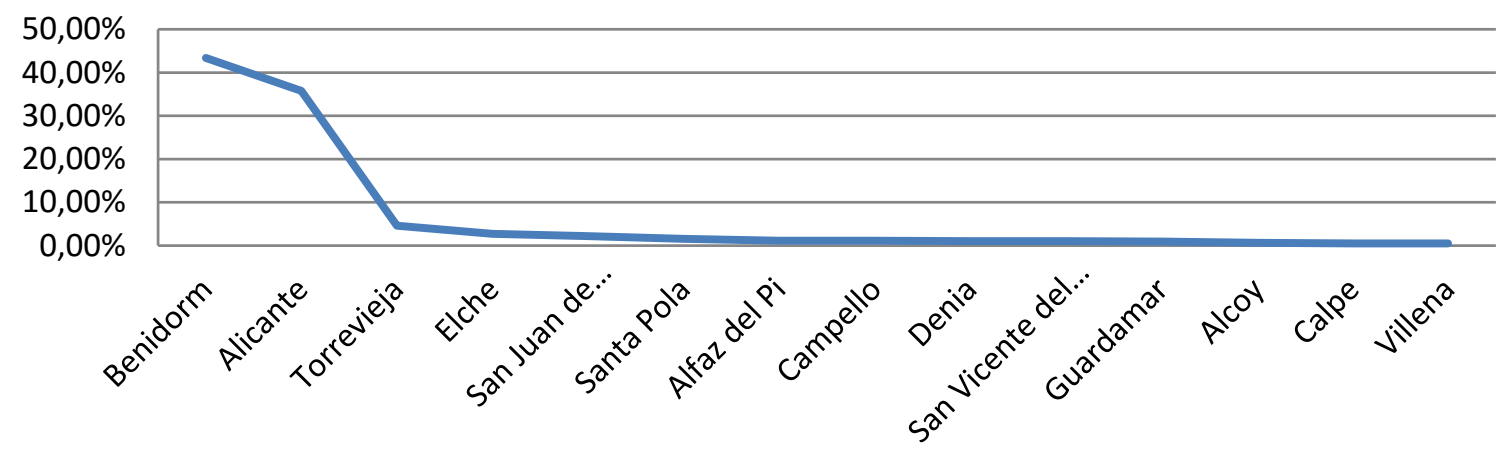

\section{VILLENA}

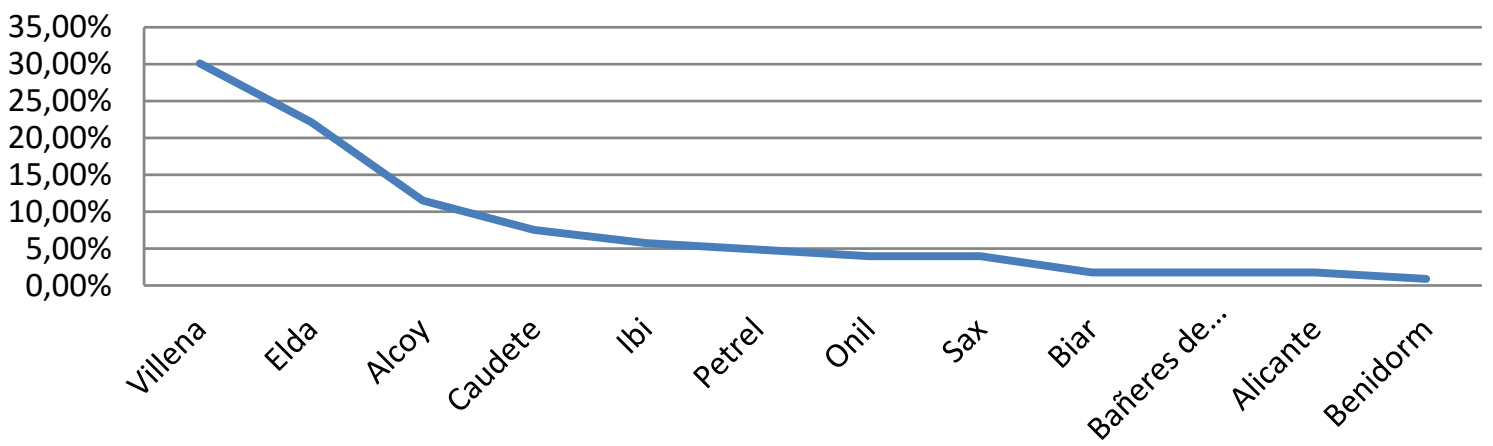

Fig. 10 y 11: destinos de la provincia de Alicante por estación.

Según las encuestas, con origen la provincia de Alicante, los motivos de viaje son más o menos constantes salvo en la época estival. En esta época del año, ganan peso los motivos "puente aéreo" (sol y playa con destinos exóticos), mientras que para Villena el motivo negocios pierde protagonismo en favor a otros más turísticos y familiares (fig. 12).

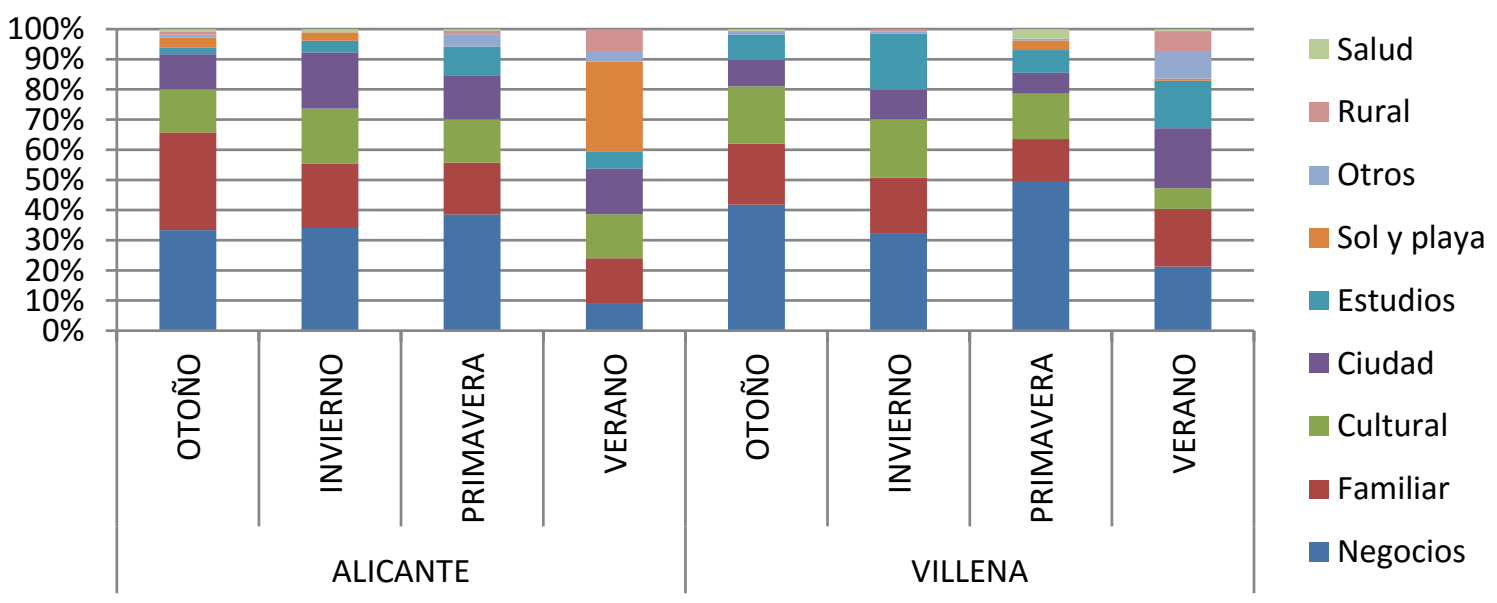

Fig. 12: motivos de viaje con origen la provincia de Alicante.

Cuando se trata del destino provincia de Alicante, no se aprecia variabilidad por estaciones 
del año, pero sí por estación ferroviaria. Para el caso de Alicante cerca del 50\% llega por motivos sol y playa mientras que para el caso de Villena la gran mayoría se reparte entre motivos familiares y negocios (fig. 13).

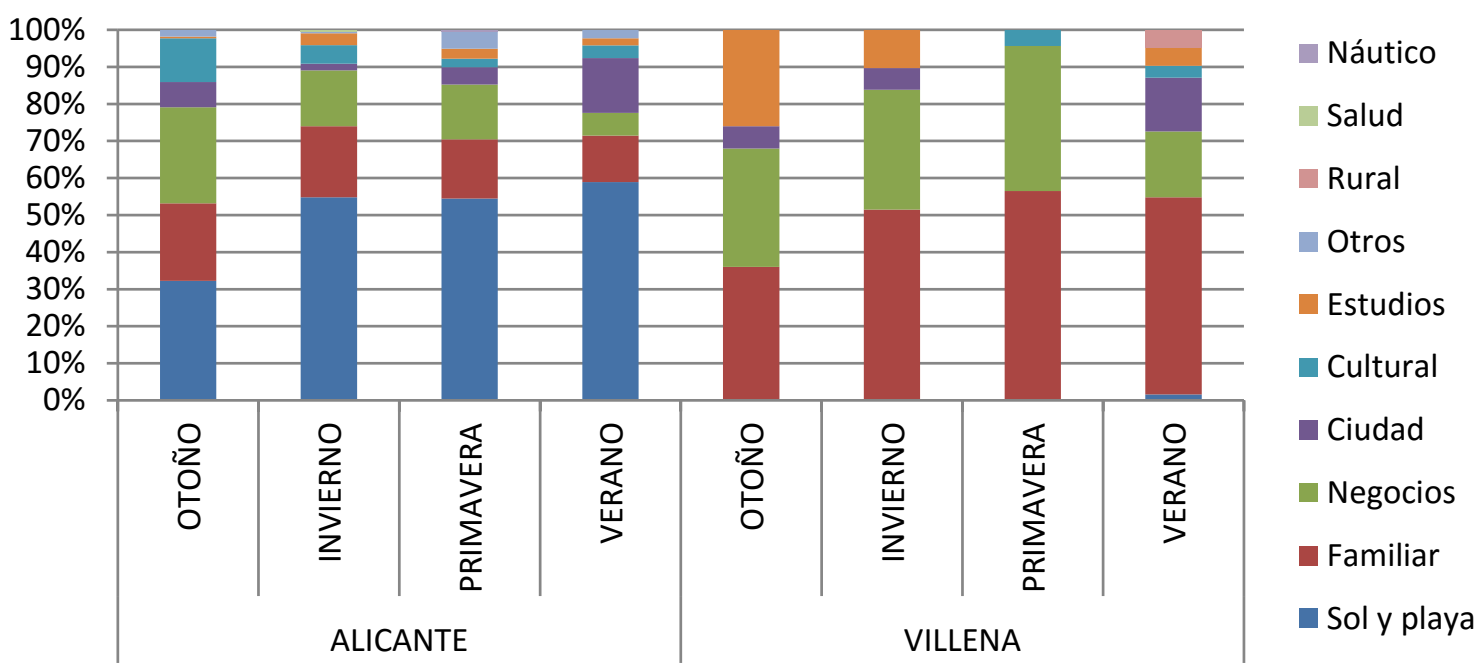

Fig. 13: motivos de viaje con destino la provincia de Alicante.

Consecuencia de estos resultados es el tipo de alojamiento escogido. En el caso de que el origen sea la provincia de Alicante, en ambas estaciones, entre el $50 \%$ y el $60 \%$ de los viajeros se aloja en hotel seguido de vivienda familiar (entre un $15 \%$ y un $30 \%$ según estación del año). Cuando el destino es la provincia de Alicante, en el caso de la estación de Alicante, el alojamiento principal es el hotel, con un 50\% en otoño y verano, llegando a un $70 \%$ en invierno y primavera. Si se trata de la estación de Villena, y en consonancia con la figura 13 , el $60 \%$ de los viajeros se alojan en una vivienda familiar.

Para finalizar, en relación a los tiempos de estancia, si el origen es la provincia de Alicante, para ambas estaciones, las estancias duran 1 ó 2 noches únicamente acentuándose en la época estival donde se presenta mayor variabilidad. En el caso de que el destino sea la provincia de Alicante, en la estación de Alicante se ha detectado que las estancias son mayoritariamente de más de 4 noches con especial importancia en las estancias de más de 9 noches. En el caso de la estación de Villena las estancias sólo superan las 3 noches en cerca de un $30 \%$ de los casos.

\section{CONCLUSIONES}

Para concluir, en primer lugar, se aprecia que no existen diferencias significativas entre estaciones cuando el destino está fuera de la provincia de Alicante, con la salvedad de los tiempos de acceso y modo escogido para llegar a la estación, siendo netamente más favorables (menos tiempo y mayor uso del transporte público) para la estación central frente a la periférica. 
Las mayores diferencias se aprecian son en los viajes cuyo destino es la provincia de Alicante. En este caso, se ha visto que la estación de Villena presenta un perfil muy distinto a la estación de Alicante, con un viajero más familiar o de motivo negocios que se dirige al territorio más cercano y poco afectado por la estacionalidad, mientras que en la estación de Alicante se encuentra a un viajero más turístico de larga estancia con destino costero y afectado por la estacionalidad, no en volumen, pero sí en edad.

\section{AGRADECIMIENTOS}

Las encuestas necesarias para este trabajo no habrían sido posibles sin la colaboración de la sociedad AVANT, José Luis Soria -subdirector de estaciones este, Adif-, Esteban Soto Jefe de la estación de Alicante, Adif-, José Antonio García Pardo, Juan Antonio Gozálbez Selles, José María Grande Torralbo, Miriam Martínez Cano, Sara Martínez Pascual y Pilar Quiles Rodríguez.

\section{REFERENCIAS}

\section{ADIF}

RENFE 